\title{
Casting dissipative compact states in coherent perfect absorbers
}

\author{
C. Danieli ${ }^{1,2, *}$ and T. Mithun $\oplus^{3,2, \dagger}$ \\ ${ }^{1}$ Max Planck Institute for the Physics of Complex Systems, Dresden D-01187, Germany \\ ${ }^{2}$ Center for Theoretical Physics of Complex Systems, Institute for Basic Science, Daejeon 34126, Republic of Korea \\ ${ }^{3}$ Department of Mathematics and Statistics, University of Massachusetts, Amherst Massachusetts 01003-4515, USA
}

(Received 2 September 2019; published 16 January 2020)

\begin{abstract}
Coherent perfect absorption (CPA), also known as time-reversed laser, is a wave phenomenon resulting from the reciprocity of destructive interference of transmitted and reflected waves. In this work we consider quasione-dimensional lattice networks which posses at least one flat band and show that CPA and lasing can be induced in both linear and nonlinear regimes of this lattice by fine-tuning non-Hermitian defects (dissipative terms localized within one unit-cell). We show that local dissipations that yield CPA simultaneously yield novel dissipative compact solutions of the lattice, whose growth or decay in time can be fine-tuned via the dissipation parameter. The scheme used to numerically visualize the theoretical findings offers a novel platform for the experimental implementation of these phenomena in optical devices.
\end{abstract}

DOI: 10.1103/PhysRevResearch.2.013054

The growing interest in non-Hermitian system is motivated by the novel and unprecedented phenomena that gain and loss terms can generate. The phenomenon of coherent perfect absorption (CPA) a notable discovery in this field which arises from the interplay of propagating waves in homogeneous media and local dissipations, and it is related to the concept of spectral singularity [1,2]. Introduced by Chong et al. in Ref. [3] and also referred as time-reversed laser, two counterpropagating coherent radiations toward a local dissipation leads to the distructive-interference of the transmitted and the scattered waves on the fine-tuning of the wave frequencies and the complex potential (see Fig. 1 for a schematic view of the process). It was also noticed that by inverting the local dissipation into a gain potential-equivalently, reversing the time-a perfect absorber can be turned into a laser where only outgoing radiations originated from the complex potential are present, while incoming ones are extinct [3]. Longhi [4] and Chong et al. [5] realized that CPA and lasing can be simultaneously achieved, yielding laser absorbers. More recently, CPA and lasing phenomena have been extended to one-dimensional nonlinear lattice chains [6,7]-where these phenomena are achieved in small propagating waves regimes-and nonlocal absorber [8]. In particular, CPA was also realized experimentally in cold atomic systems [7]. An important open frontier is

\footnotetext{
*cdanieli@pks.mpg.de

†mthudiyangal@umass.edu
}

Published by the American Physical Society under the terms of the Creative Commons Attribution 4.0 International license. Further distribution of this work must maintain attribution to the author(s) and the published article's title, journal citation, and DOI. to extend CPA to lattices which support multiple Bloch bands with distinct spectral properties. This may induce additional novel phenomena in lattice in presence of a non-Hermitian source. Moreover, it would substantially extend the applicability and the experimental implementation of perfect absorbers and lasers.

In the recent years a growing amount of attention has been received by translationally invariant networks which simultaneously support propagating waves and compact localization. These systems - commonly referred as flat band networksare characterized by the existence of at least one dispersionless (flat) spectral band $[9,10]$. The corresponding eigenmodes of the flat band are typically strictly compact in space-hence dubbed compact localized states (CLS) - and they arise due to destructive interference. Notable theoretical advances for these systems includes systematic generator schemes [11-14] localization phenomena due to onsite perturbations [15-18], and the existence of compact breathers in flat band networks with Kerr nonlinearity [19-21]. Moreover, these systems have been realized experimentally in several set-ups, including photonics lattices [22-24] to exciton-polariton condensates [25] and ultra cold atoms [26], among others. These findings highlight how flat band networks are ideal set-ups to study and implement novel and highly relevant phenomena in condensed matter physics.

Both the CLS and CPA are wave phenomena resulting from destructive interference. However, the question "Can these phenomena be embedded in a single device?" has not yet been tackled. In this work we address this yet unanswered question by considering flat band networks in the presence of Kerr nonlinearity and complex non-Hermitian dissipative terms localized within one unit-cell. We then analytically prove and numerically verify that the chosen dissipations can induce CPA phenomenon out of dispersive waves, as well as induce nonpropagating excitations localized at the dissipative sites out of the flat band states. 


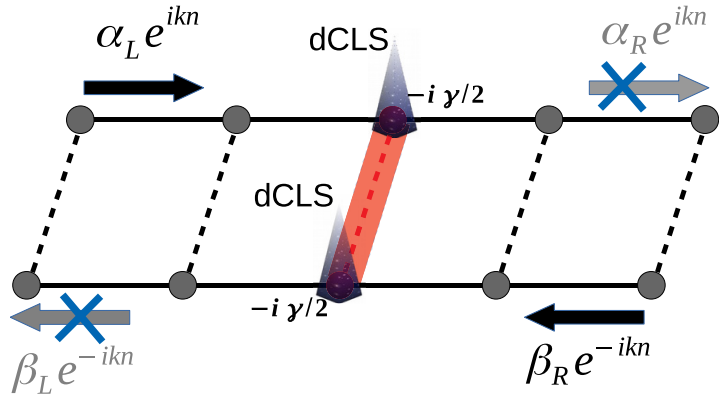

FIG. 1. Schematic representation of CPA: $\alpha_{L}, \beta_{R}$ and $\alpha_{R}, \beta_{L}$ control the incident and the reflected waves, respectively; the red area represents the localized dissipation. The trigones represent the dissipative compact state (dCLS).

Let us consider the equations of motion of a network with $v$ sites per unit-cell,

$$
i \dot{\Psi}_{n}=-H_{0} \Psi_{n}-H_{1} \Psi_{n+1}-H_{1}^{\dagger} \Psi_{n-1}-\frac{i \gamma}{2} V \Psi_{0} \delta_{n, 0},
$$

represented by the time-dependent complex vector $\Psi_{n} \in \mathbb{C}^{v}$. Here $H_{0}$ and $H_{1}$ are real square matrices of size $v$. The dissipation is localized within the unit-cell at $n=0$, where $\gamma>0$ and the square matrix $V$ encodes the gain and loss terms of the non-Hermitian terms-non-Hermitian defects. The steady-state solution $\Psi_{n}=U_{n} e^{-i \mu t}$ of Eq. (1) yields the eigenvalue problem

$$
\mu U_{n}=-H_{0} U_{n}-H_{1} U_{n+1}-H_{1}^{\dagger} U_{n-1}-\frac{i \gamma}{2} V U_{0} \delta_{n, 0} .
$$

For $\gamma=0$ (no dissipation), the Bloch solution $U_{n}=e^{i k n} \Phi_{k}$ turns Eq. (2) to $\mu \Phi_{k}=B(k) \Phi_{k}$ where the matrix $B(k) \equiv$ $-H_{0}-e^{i k} H_{1}-e^{-i k} H_{1}^{\dagger}$ depends on the wave vector $k$. This resulting eigenvalues of the matrix $B(k)$ yield the band structure formed by $v$ Bloch bands $\Omega=\left\{\mu_{j}(k)\right\}_{j=1}^{v}$ of Eq. (1). In this work we consider systems which contains at least one flat band.

Let us consider the following ansatz of solution for Eq. (1):

$$
U_{n}=\Phi_{j} \begin{cases}\alpha_{L} e^{i k n}+\beta_{L} e^{-i k n} & n<0 \\ u_{0} & n=0 \\ \alpha_{R} e^{i k n}+\beta_{R} e^{-i k n} & n>0\end{cases}
$$

where $\Phi_{j}$ is the eigenvector of $B(k)$ correspondent to the dispersive band $\mu_{j}$. Hence, $\alpha_{L}$ and $\beta_{R}$ control the incident waves from the left $(L)$, while $\alpha_{R}$ and $\beta_{L}$ the reflected wave from the right $(R)$. In this framework, CPA implies to fine-tune the wave vector $k$ in Eq. (3) with respect to the dissipation components $\{\gamma, V\}$ in order to admit the incident radiations $\alpha_{L}, \beta_{R}$ and annihilate the reflected radiations $\alpha_{R}, \beta_{L}$ as represented in Fig. 1. In general, different choices of the dissipation matrix $V$ may be considered to induce CPA, depending on the dispersive wave considered. Certain choices of $V$ can both (i) induce fine-tuned CPA from counterpropagating dispersive waves and (ii) preserve the CLS of the flat band which overlaps with the dissipative cell, inducing nonpropagating localized excitations centered at the dissipative unitcell whose amplitudes either decay exponentially/grow exponentially/stay constant in time depending on the dissipation parameters. Following Refs. [6,7], (i) in general holds true
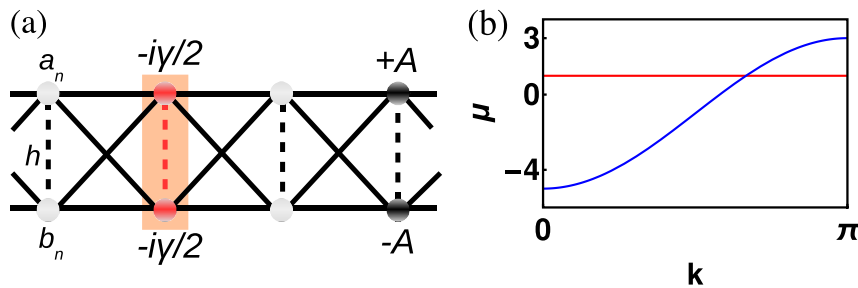

FIG. 2. (a) Profile of the cross-stitch lattice. (b) Band structure for $h=1$.

when considering the Kerr nonlinearity $g \mathcal{F}\left(\Psi_{n}\right) \Psi_{n}$ in Eq. (1) where $\mathcal{F}\left(\Psi_{n}\right)=\sum_{j}\left|\psi_{n}^{j}\right|^{2} e_{j} \otimes e_{j}$ with $\left\{e_{j}\right\}$ canonical basis of $\mathbb{R}^{v}$. However, (ii) depends on whether the linear CLS can be continued as compact time-periodic solution (breather) in the nonlinear regime of the lattice [21].

To illustrate these statements, we employ a simple onedimensional two-band network $v=2$ with $\Psi_{n}=\left(a_{n}, b_{n}\right)^{T}$, as shown in Fig. 2(a). The matrices $H_{0}, H_{1}$ in Eq. (1) that define the cross-stitch lattice (CS) are

$$
H_{0}=\left(\begin{array}{ll}
0 & h \\
h & 0
\end{array}\right), \quad H_{1}=\left(\begin{array}{ll}
1 & 1 \\
1 & 1
\end{array}\right), \quad V=V_{1} \equiv\left(\begin{array}{ll}
1 & 0 \\
0 & 1
\end{array}\right) \text {. }
$$

For $\gamma=0$, the linear CS network possesses one dispersive band $\mu_{1}(k)=-h-4 \cos k$ and one flat band $\mu_{2}=h$, as shown in Fig. 2(b) for $h=1$.

To fine-tune CPA in the CS network, in Eq. (3) we consider the normalized eigenvector $\Phi_{1}=\frac{1}{\sqrt{2}}(1,1)^{T}$ correspondent to the model's dispersive band $\mu_{1}(k)$. Considering the local symmetry of both the chain and $\Phi_{1}$, it naturally follows to choose a symmetric diagonal dissipative matrix $V=V_{1}$ in Eq. (4). This choice induce the CPA condition for a given $k$-see Appendix A for details,

$$
\gamma_{*}=8 \sin k .
$$

Moreover, CPA occurs also in the nonlinear regime $g \neq 0$ of the lattice at the same condition Eq. (5) in perturbative regimes (small propagating waves regime) - as detailed in Appendix A.

We visualize numerically this theoretical prediction in the nonlinear CS network with nonlinear strength $g=1$ by considering two counterpropagating Gaussian beams [27] centered far from the dissipative unit-cell $n=0$ at unit-cells $n_{L} \ll 0$ and $n_{R} \gg 0$ respectively, with $n_{L}=-n_{R}$. Written in components $\Psi_{n}=\left(a_{n}, b_{n}\right)^{T}$, the Gaussian beams at $t=0 \mathrm{read}$

$$
a_{n}=b_{n}=P_{0} e^{-\alpha\left(n-n_{R}\right)^{2}} e^{-i k\left(n-n_{R}\right)}+P_{0} e^{-\alpha\left(n-n_{L}\right)^{2}} e^{i k\left(n-n_{L}\right)},
$$

where $P_{0}=0.01$ is the amplitude and $\alpha=0.0075$ is the inverse width of the excitation-see Appendix B for details. For $k=1$ in Eq. (6), the CPA condition Eq. (5) yields $\gamma_{*} \approx 6.73$. In Figs. 3(a) and 3(b) we show the time evolution of the local densities $S_{n}=\left|a_{n}\right|^{2}+\left|b_{n}\right|^{2}$ for $\gamma=\gamma_{*} \approx 6.73$ [Fig. 3(a)] and $\gamma=1$ [Fig. 3(b)]. At the CPA condition [Fig. 3(a)] the two incoming radiations are fully absorbed at the dissipative cell $n=0$ (signaled with the blue horizontal line), and no propagating 

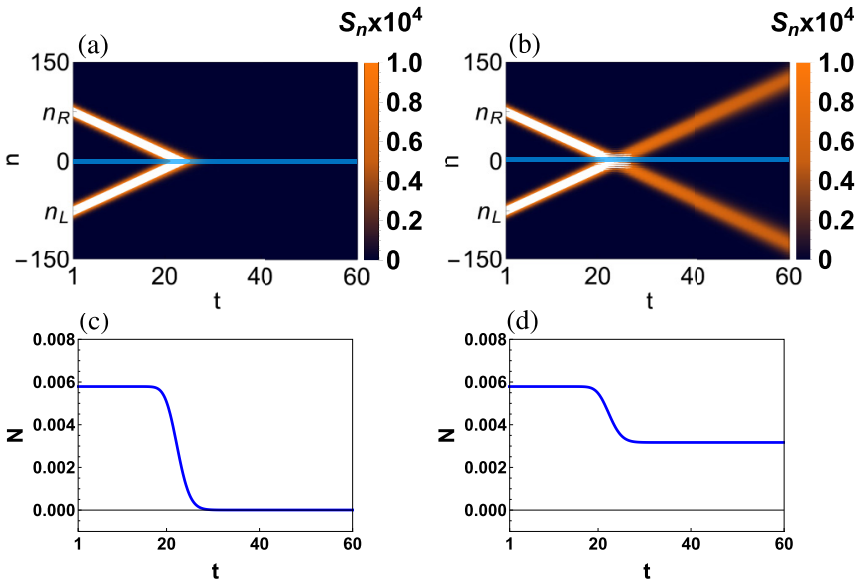

FIG. 3. Dispersive case $\Phi_{1}$ : Time evolution of $S_{n}$ (a) and $N$ (c) for $\gamma=6.73 \approx \gamma_{*}$ and $S_{n}$ (b) and $N$ (d) for $\gamma=1$. Here $k=1$ and $g=1$, while $n_{R}=75$ and $n_{L}=-75$. The blue horizontal lines signal the dissipative unit-cell at $n=0$.

radiation follows. Instead, away from the CPA condition [Fig. 3(b)] the two incoming radiations are only partially absorbed and propagating radiations survive. This complete and partial absorption is further visualized in Figs. 3(c) and $3(d)$, respectively, where we show the time evolution of the total norm $N=\sum_{n} S_{n}$.

In Fig. 4(a) we show the unabsorbed total norm $N$ at time $t=60$ computed for different values of the dissipation strength $\gamma$. This is further detailed in the inset, where we show the percentage of remaining norm $\%=100 \frac{N(t=60)}{N(t=0)}$ versus $\gamma$. The minimum of the curve lies to $\gamma_{*}$, denoted by the vertical orange line, where $\% \approx 0$. We can additionally observe that a significant part of the curve lies below the horizontal green line (line denoting 1\%). This indicates that there exists a non-negligible neighborhood of $\gamma_{*}$ where any $\gamma$ chosen within such interval leaves less than $1 \%$ of unabsorbed incoming radiations.

Due to destructive interference, the normalized eigenvector $\Phi_{2}=\frac{1}{\sqrt{2}}(1,-1)^{T}$ of $B(k)$ correspondent to the flat band $\mu_{2}$ of the CS lattice introduced in Eq. (3) does not yield propagating waves. Indeed, considering $a_{n}=-b_{n}$ in Eq. (6) with $n_{R} \gg 0$ and $n_{L} \ll 0$ yields two nonpropagating Gaussian excitations centered at $n_{R}$ and $n_{L}$, respectively, as shown in Fig. 5(a). This also persists for $n_{L}=0=n_{R}$, as shown in Fig. 5(b). However, the local density $S_{n}$ at $n=0$ of the nonpropagating excitation
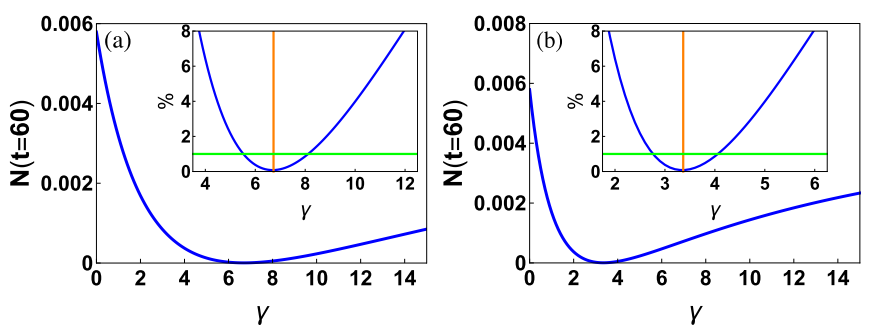

FIG. 4. Total norm $N$ at $t=60$ versus $\gamma$ for $V_{1}$ (a) and $V_{2}$ for $\delta=1$ (b). Inset: Percentage (\%) at $t=60$, with $\gamma_{*}$ (orange); $1 \%$ of the norm (green). Here $h=1$ and $g=1$.

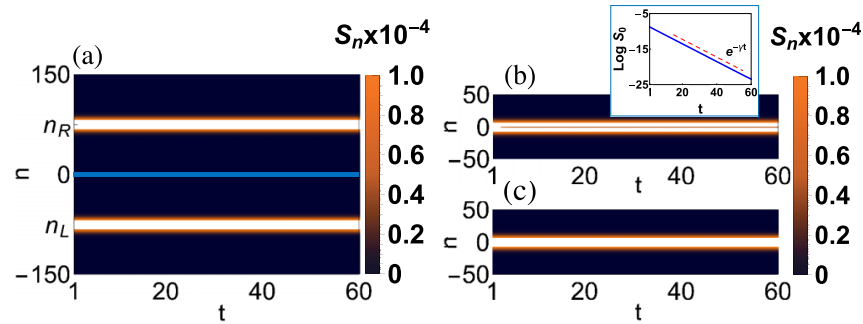

FIG. 5. Flat band case $\Phi_{2}$ : (a) Time evolution of $S_{n}$ for $\gamma=0.25$ and $\delta=0$ while $n_{R}=75$ and $n_{L}=-75$. (b) Same as in (a) with $n_{R}=0=n_{L}$. Inset: Time evolution of $S_{0}$. (c) Time evolution of $S_{n}$ for $\gamma=0.25, \delta=1$ with $n_{R}=0=n_{L}$. Here $g=1$ and $k=1$.

decays exponentially in time as $S_{0} \sim e^{-\gamma t}$, as shown in the inset of Fig. 5(b).

The emergence of the dissipative nonpropagating excitation at $n=0$ shown in Fig. 5(b) follows from the fact that the dissipation $V_{1}$ in Eq. (4) is symmetric between the two sublattices and therefore it does not break the CLS located at the unit-cell $n=0$, with the state indicated with black dots in Fig. 2(a), but it turns its frequency complex, $\mu=h-i \gamma / 2$. Hence, this CLS turns dissipative. An analogous fate occurs in the nonlinear regime of the CS lattice. Indeed, as discussed in Ref. [21], Kerr nonlinearity preserves destructive interference, and the dissipative CLS at $n=0$ with amplitude $A$

$$
U_{n, 0}(t)=A\left(\begin{array}{c}
1 \\
-1
\end{array}\right) \delta_{n, 0} e^{-i \Omega t}
$$

becomes a dissipative solution of the lattice with renormalized frequency $\Omega=h+g A^{2}-i \gamma / 2$. In both linear and nonlinear regime of the CS, the local density of the compact solution decays exponentially in time $S_{0}(t) \sim e^{-\gamma t}$ due to the imaginary term $-i \gamma / 2$ in their frequency. This behavior in time can be controlled by employing a second dissipation matrix $V_{2}$ in Eq. (4) for $\delta \in \mathbb{R}$,

$$
V=V_{2} \equiv\left(\begin{array}{ll}
1 & \delta \\
\delta & 1
\end{array}\right)
$$

The frequency of the compact dissipative solution Eq. (7) reads $\Omega=h+g A^{2}-i \gamma(1-\delta) / 2$. Hence $S_{0}(t) \sim e^{-\gamma(1-\delta) t}$, which recalling that for any $\gamma>0$ implies that the local density $S_{0}$ of the compact solution decays exponentially for $\delta<1$, grows exponentially for $\delta>1$ and stay constant for $\delta=1$. This consequently implies the existence of a nonpropagating excitations located at the dissipative unit-cell with the amplitude at the dissipative cell $n=0$ that decays, grows, or stays constant with time according to the dissipation parameters, as shown in Fig. 5(c) for $\delta=1$.

This second dissipative matrix $V_{2}$ in Eq. (8) not only allows for fine control of the time behavior of the compact flat band solutions, but it also induces CPA with condition

$$
\gamma_{*}=\frac{8}{1+\delta} \sin k
$$

that generalizes Eq. (5); see Appendix A for details. This prediction has been numerically confirmed in Fig. 4(b) in the same framework as for $V_{1}$. In particular, we can again observe in the inset that while CPA is achieved at $\gamma_{*} \approx 3.115-\mathrm{a}$ 

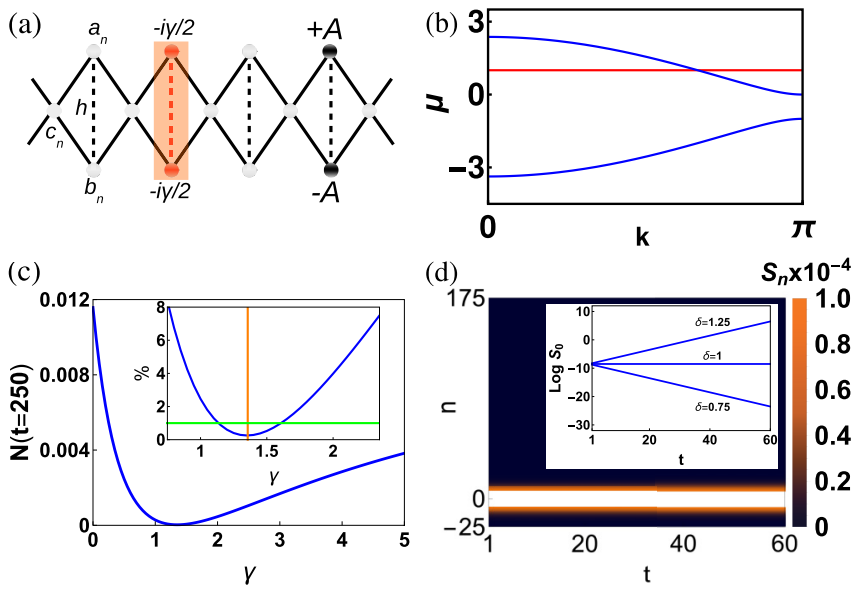

FIG. 6. (a) Profile of the diamond chain. (b) Band structure. (c) Total norm $N$ at $t=250$ versus $\gamma$ for $\delta=1$. Inset: Percentage (\%) at $t=250$, with $\gamma_{*}$ (orange); $1 \%$ of the norm (green). (d) Time evolution of $S_{n}$ for $\gamma=1, \delta=1$. Inset: Time evolution of $S_{0}$ for $\delta=1.25$ (top), $\delta=1$ (middle), and $\delta=0.75$ (bottom) with $\gamma=1$. Here $g=1$ and $h=1$.

condition obtained from Eq. (9) with $k=1$ and $\delta=1-\mathrm{a}$ nontrivial neighborhood of $\gamma_{*}$ exists where less than $1 \%$ of the incoming beams' total norm is unabsorbed.

Remarkably, this procedure works and CPA can be obtained in a plethora of other flat band topologies. For example, the diamond chain, whose profile is shown in Fig. 6(a) and the equations of motion written in components $\Psi_{n}=\left(a_{n}, b_{n}, c_{n}\right)^{T}$ with local dissipation controlled by the parameters $\gamma, \delta$ and in the presence of Kerr nonlinearity are shown below

$$
\begin{aligned}
& i \dot{a}_{n}=-c_{n}-c_{n+1}-h b_{n}-\frac{i \gamma}{2}\left(a_{0}+\delta b_{0}\right) \delta_{n, 0}+g a_{n}\left|a_{n}\right|^{2} \\
& i \dot{b}_{n}=-c_{n}-c_{n+1}-h a_{n}-\frac{i \gamma}{2}\left(b_{0}+\delta a_{0}\right) \delta_{n, 0}+g b_{n}\left|b_{n}\right|^{2} \\
& i \dot{c}_{n}=-a_{n-1}-a_{n}-b_{n-1}-b_{n}+g a_{n}\left|a_{n}\right|^{2} .
\end{aligned}
$$

For $g=0$ and $\gamma=0$, Eq. (10) possesses two dispersive bands, $\mu_{1,2}(k)=\left(-h \pm \sqrt{h^{2}+16+16 \cos k}\right) / 2$, and one flat band, $\mu_{3}=h$ [Fig. 6(b)]. This model (also called rhombic lattice) has been employed to theoretically study the impact of nonHermitian hopping [28], magnetic field [29-31], and electric fields [32] on flat band networks, as well as to experimentally realize compact localized states [33], study CLS in driven photonic flat band lattices [34], and experimentally observe Aharonov-Bohm caging effect [35].

Via the transfer matrix formalism, CPA can be fine-tuned in the diamond chain considering the eigenmodes $\phi_{1,2}=$ $\left(1,1, \pm \sqrt{2} e^{-k / 2}\right)^{T} / 2$ in the ansatz Eq. (3) correspondent to the dispersive bands $\mu_{1,2}$. For both bands, CPA occurs with the condition

$$
\gamma_{*}=\frac{4 \sqrt{2}}{1+\delta} \sin \frac{k}{2},
$$

which still holds in the nonlinear regime $g \neq 0$ of the diamond chain Eq. (10).

This theoretical prediction can be achieved with two counterpropagating Gaussian beams located at $n_{L} \ll 0$ and
$n_{R} \gg 0$, respectively, with $n_{L}=-n_{R}$, which in the diamond chain case are $b_{n}=a_{n}=P_{0} e^{-\alpha\left(n-n_{R}\right)^{2}} e^{-i k\left(n-n_{R}\right)}+$ $P_{0} e^{-\alpha\left(n-n_{L}\right)^{2}} e^{i k\left(n-n_{L}\right)}$ and $c_{n}= \pm \sqrt{2} e^{-k / 2} a_{n}$; see Appendix B for details. For $k=1$ and $\delta=1$, Eq. (11) predicts that CPA is achieved for $\gamma_{*} \approx 1.36$, which is visualized analogously with the CS in Fig. 6(c). On the other hand, the flat band eigenvector $\Phi_{3}=\frac{1}{\sqrt{2}}(1,-1,0)^{T}$ of $B(k)$ of the diamond chain induces a nonpropagating Gaussian excitation located at $n=0$ [Fig. 6(d)]. The reason for this phenomenon follows from the fact that the chosen dissipation does not lift the linear CLS at $n=0$-indicated with black dots in Fig. 6(a) — but it only alters its frequency and makes it dissipative. This still holds in the nonlinear regime $g \neq 0$ of Eq. (10) since the linear CLS locate at the unit-cell $n=0$ can be continued as compact breather with frequency $\Omega=h+g A^{2}-i \gamma(1-\delta) / 2$ [21]. This yields a precise control of the time behavior of the breather density $S_{0}$, namely $S_{0}$ decays exponentially for $\delta<1$, grows exponentially for $\delta>1$, and remains constant for $\delta=1$-as shown in the inset of Fig. 6(d).

By choosing certain flat band geometries, we showed that local dissipations can be arranged to induce CPA and at the same time preserve the compact solution that exists at the dissipative unit-cell. This notably relates to the local symmetry in the chosen dissipative terms (matrix $V$ ) as shown in Appendix A. If this symmetry in $V$ is broken, then the destructive interference that yields CLS is locally lifted and the system does not admit dissipative compact statesalthough it still can support CPA. The current setting of a single dissipative unit-cell in Eq. (1) prevents simultaneous achievement of CPA and dissipative CLS in networks whose compact states occupy at least $U \geqslant 2$ unit-cells-e.g., the Lieb lattice [36] — since the partial overlap between CLS and dissipation lifts destructive interference. Hence, the existence of dissipative CLS requires the local dissipation to span over at least $U$ unit-cells with a profile which respects the CLS symmetries. Such dissipative CLS may also become dissipative compact solutions of the nonlinear regime-in agreement with Ref. [21] —as well as their time behavior (exponential growth, decay, or being constant) can be controlled by tuning the dissipation parameters, in analogy with what we herewith reported. Moreover, following Refs. [6,7], we observe that the absorption conditions in Eqs. (5), (9), and (11) can be reversed into lasing conditions by permuting the sign of the prefactor $i \gamma / 2$ of the dissipative terms $V$ in Eq. (1) to positive $+i \gamma / 2$. This namely implies that in Eq. (3) the incoming radiations $a_{L}, b_{R}$ are annihilated and only outgoing radiations $a_{R}, b_{L}$ emerge from the non-Hermitian unit-cell.

To summarize, we have shown that local dissipations in lattice networks can yield simultaneously two wave phenomena: coherent perfect absorption and the existence of dissipative compact localized states. Both phenomena are the result of destructive wave interference, and we have shown that they can be embedded in a single device which posseses both dispersive and flat bands by engineering the local dissipations. In a broader perspective, our results first usher the existence and the study of compact dissipative breathers into nonlinear non-Hermitian media (see Ref. [37] for a recent survey on dissipative discrete breathers) which may additionally account for Ghost states in the case of $\mathcal{P} \mathcal{T}$-symmetric nonlinear 
networks [38]. Additionally, it is interesting to note the analogy between the dissipative compact solutions induced by symmetric local dissipation with the bound states in the continuum waves studied in Ref. [39]. Second, these findings pave the way to accomplish CPA and lasing phenomena in multiband networks supporting propagating waves. These include optical and photonic systems as well as excitonpolaritons and microwave systems, all systems where flat band networks have been studied and experimentally realized. Moreover the Gaussian beams set-up herewith employed to visualize our theoretical findings offers a novel and powerful scheme to experimentally realize CPA in order to fabricate switches [40], interferometers [41], and logic elements [42] in several physically relevant frameworks, particularly nonlinear optical and photonic lattice networks.

The authors acknowledge financial support from the Institute for Basic Science Project Code No. IBS-R024-D1. We thank S. Flach, A. Andreanov, and P. Kevrekidis for helpful discussions.

\section{APPENDIX A: COHERENT PERFECT ABSORPTION CONDITIONS}

\section{Transfer matrix method}

Let us recap the ansatz

$$
U_{n}=\Phi_{j} \begin{cases}\alpha_{L} e^{i k n}+\beta_{L} e^{-i k n} & n<0 \\ u_{0} & n=0 \\ \alpha_{R} e^{i k n}+\beta_{R} e^{-i k n} & n>0\end{cases}
$$

For $\phi_{j}$ the eigenmode of the matrix Bloch $B(k)$ correspond to one dispersive band $\mu_{j}$ of a multiband network. We define

$$
a_{L}=\alpha_{L} \Phi_{j}, \quad a_{R}=\alpha_{R} \Phi_{j}, \quad b_{L}=\beta_{L} \Phi_{j}, \quad b_{R}=\beta_{R} \Phi_{j},
$$

where $a_{L}$ and $b_{R}$ are the incident wave amplitudes from left $(L)$ and right $(R)$, while $a_{R}$ and $b_{L}$ are the reflected wave amplitudes. We then introduce the transfer matrix $M$, defined as

$$
\left(\begin{array}{l}
a_{R} \\
b_{R}
\end{array}\right)=M\left(\begin{array}{l}
a_{L} \\
b_{L}
\end{array}\right), \quad \text { where } \quad M=\left[\begin{array}{ll}
M_{11}(k) & M_{12}(k) \\
M_{21}(k) & M_{22}(k)
\end{array}\right] .
$$

In this case, the transfer matrix $M$ is a square matrix of size $2 v$ and the components $M_{i, j}(k)$ blocks of side $v$.

Following Ref. [7], CPA conditions can be derived via the transfer matrix $M$. CPA occurs when there exist nonzero incoming radiations $a_{L}$ and $b_{R}$, but the outgoing radiations $a_{R}$ and $b_{L}$ are zero. In Eq. (A3), this translates into finding the $k_{*}$ such that $M_{11}\left(k_{*}\right)=\mathbb{O}_{\nu}[6,7]$, since

$$
\left(\begin{array}{c}
0 \\
b_{R}
\end{array}\right)=\left[\begin{array}{l}
M_{11}\left(k_{*}\right) a_{L} \\
M_{21}\left(k_{*}\right) a_{L}
\end{array}\right], \Leftrightarrow\left\{\begin{array}{l}
M_{11}\left(k_{*}\right)=\mathbb{O}_{v} \\
b_{R}=a_{L}
\end{array}\right.
$$

and $M_{21}\left(k_{*}\right)=1$. Here $\left(k_{*}\right)^{2}$ represents a time-reversed spectral singularity. Let us remark that reversing the conditionincoming radiations $a_{L}$ and $b_{R}$ are zero, but the outgoing radiations $a_{R}$ and $b_{L}$ are nonzero-yields lasing condition
[5]. In Eq. (A3), this translates into finding the $k_{*}$ such that $M_{22}\left(k_{*}\right)=\mathbb{O}_{v}[6,7]$.

\section{Cross-stitch: Linear regime}

The cross-stitch lattice is a $v=2$ bands problem,

$$
\begin{aligned}
\mu U_{n} & =-H_{0} U_{n}-H_{1} U_{n+1}-H_{1}^{\dagger} U_{n-1}-i \frac{\gamma}{2} V U_{0} \delta_{n, 0} ; \\
H_{0} & =\left(\begin{array}{ll}
0 & h \\
h & 0
\end{array}\right), \quad H_{1}=\left(\begin{array}{ll}
1 & 1 \\
1 & 1
\end{array}\right),
\end{aligned}
$$

with one dispersive band $\mu_{1}(k)=-h-4 \cos k$, whose correspondent eigenvector is $\Phi_{1}=\frac{1}{\sqrt{2}}(1,1)^{T}$. In this case, we choose two type of dissipations matrix $V$ defined as

$$
V_{1}=\left(\begin{array}{ll}
1 & 0 \\
0 & 1
\end{array}\right) \text { and } V_{2}=\left(\begin{array}{ll}
1 & \delta \\
\delta & 1
\end{array}\right)
$$

a. Case 1: $V_{1}$. For the cross-stitch lattice $H_{1}=H_{1}^{\dagger}$. Equation (A5) at $n=\{0, \pm 1\}$ yields

$$
\begin{gathered}
{\left[\mu_{1} \mathbb{I}_{2}+H_{0}+i \frac{\gamma}{2} V_{1}\right] U_{0}} \\
=-H_{1}\left[a_{R} e^{i k}+b_{R} e^{-i k}+a_{L} e^{-i k}+b_{L} e^{i k}\right], \\
{\left[\mu_{1} \mathbb{I}_{2}+H_{0}\right]\left[U_{1}+U_{-1}\right]=-H_{1}\left(U_{2}+U_{-2}\right)-2 H_{1} U_{0},} \\
{\left[\mu_{1} \mathbb{I}_{2}+H_{0}\right]\left[U_{1}-U_{-1}\right]=-H_{1}\left(U_{2}-U_{-2}\right) .}
\end{gathered}
$$

From Eq. (A2), we sort Eqs. (A7), (A8), and (A9) in terms of $\Phi_{1}$. Recalling the identities $H_{0} \Phi_{1}=h \Phi_{1}, H_{1} \Phi_{1}=$ $2 \Phi_{1}$, and $\left[\mu_{1} \mathbb{I}_{2}+H_{0}\right] \Phi_{1}=-4 \cos k \Phi_{1}$, Eqs. (A8) and (A9) reduce to

$$
\begin{gathered}
{\left[\alpha_{R}+\beta_{R}+\alpha_{L}+\beta_{L}\right] \Phi_{1}=H_{1} U_{0},} \\
{\left[\alpha_{R}+\beta_{R}\right] \Phi_{1}=\left[\alpha_{L}+\beta_{L}\right] \Phi_{1} .}
\end{gathered}
$$

Since the identity $\left[\mu_{1} \mathbb{I}_{2}+H_{0}+i \frac{\gamma}{2} V_{1}\right] \Phi_{1}=\left(-4 \cos k+\frac{i \gamma}{2}\right) \Phi_{1}$, from Eq. (A7) it follows

$$
U_{0}=-\frac{2}{-4 \cos k+i \frac{\gamma}{2}}\left[\alpha_{R} e^{i k}+\beta_{R} e^{-i k}+\alpha_{L} e^{-i k}+\beta_{L} e^{i k}\right] \Phi_{1} .
$$

In Eq. (A10), this ultimately results into the equality

$$
\begin{aligned}
& {\left[\alpha_{R}\left(4 \sin k+\frac{\gamma}{2}\right)-\beta_{R}\left(4 \sin k-i \frac{\gamma}{2}\right)\right] \Phi_{1}} \\
& \quad=\left[\alpha_{L}\left(4 \sin k-\frac{\gamma}{2}\right)-\beta_{L}\left(4 i \sin k+i \frac{\gamma}{2}\right)\right] \Phi_{1} .
\end{aligned}
$$

Since $\Phi_{1}=\frac{1}{\sqrt{2}}(1,1)^{T}$, we just refer to both Eqs. (A11) and (A13) as equations of scalar numbers.

These two conditions ultimately yield the entrees of the transfer matrix $M$ Eq. (A4)

$$
\begin{aligned}
M_{11} & =\frac{8 \sin k-\gamma}{8 \sin k} \mathbb{I}_{2} & M_{12} & =\frac{-\gamma}{8 \sin k} \mathbb{I}_{2} \\
M_{21} & =\frac{\gamma}{8 \sin k} \mathbb{I}_{2} & M_{22} & =\frac{8 \sin k+\gamma}{8 \sin k} \mathbb{I}_{2} .
\end{aligned}
$$



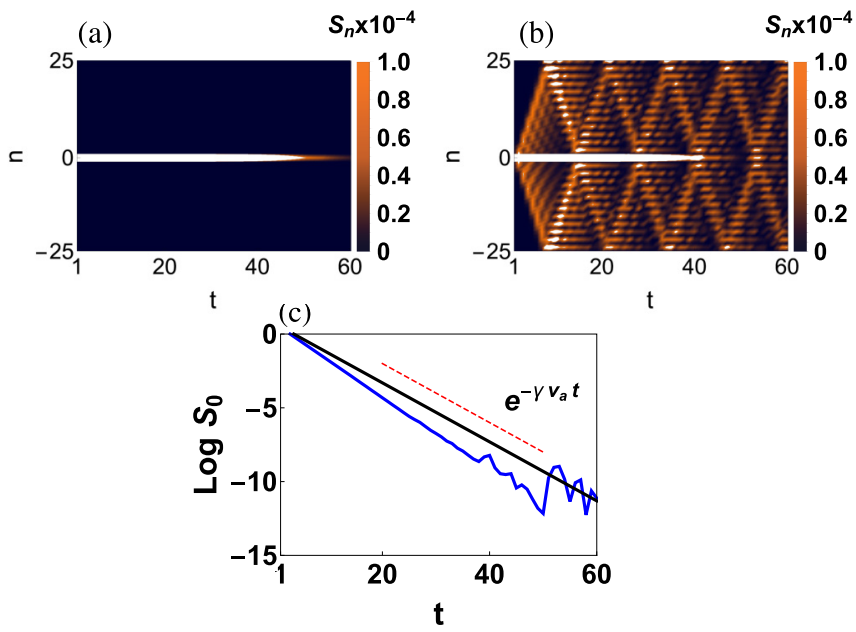

FIG. 7. (a) Time evolution of $S_{n}$ for $v_{a}=1=v_{b}$. (b) Time evolution of $S_{n}$ for $v_{a}=1$ and $v_{b}=1.25$. (c) Time evolution of $S_{0}$ for $v_{a}=1=v_{b}$ (black) and for $v_{a}=1$ and $v_{b}=1.25$ (blue). The red dashed line indicates the exponential decay $S_{0} \sim e^{-\gamma v_{a} t}$.

Imposing $M_{11}(k)=\mathbb{O}_{2}$ yields the CPA condition

$$
\gamma_{*}=8 \sin k \Leftrightarrow \quad k_{*}=\arcsin \frac{\gamma}{8} .
$$

b. Symmetry-imposed coexistence of CLS and CPA. Let us consider the CS lattice with diagonal elements $v_{a}, v_{b}$ in the matrix $V_{1}$ in Eq. (A6)-Eq. (4) of the main text,

$$
\begin{aligned}
& \mu a_{n}=-a_{n-1}-a_{n+1}-b_{n-1}-b_{n+1}-h b_{n}-\frac{i \gamma}{2} v_{a} a_{0} \delta_{n, 0} \\
& \mu b_{n}=-b_{n-1}-b_{n+1}-a_{n-1}-a_{n+1}-h a_{n}-\frac{i \gamma}{2} v_{b} b_{0} \delta_{n, 0} .
\end{aligned}
$$

For $v_{a}=v_{b}$, the case in the former section is obtained, which leads to CPA as well as to dissipative CLS with frequency $\Omega=h-i \gamma / 2$. For $v_{a} \neq v_{b}$, the two components of the compact state $a_{0}, b_{0}$ decay at different rates, namely $\left|a_{0}\right|^{2} \sim$ $e^{-\gamma v_{a} t}$ and $\left|b_{0}\right|^{2} \sim e^{-\gamma v_{b} t}$. This lifts destructive interference and consequently destroy the compact state. This is shown in Fig. 7, where the time evolution of $S_{n}$ is shown for the CLS with $\gamma=0.2$ and $v_{a}=1=v_{b}$ in Fig. 7(a) and $v_{a}=1$ while $v_{b}=1.25$ in Fig. 7(b), confirming that in the former case compactness is preserved and in the latter case compactness is lost. In Fig. 7(c) we show how the exponential decay of $S_{0} \sim e^{-\gamma v_{a} t}$ for $v_{a}=v_{b}$ (black curve) is distorted and lost for $v_{a} \neq v_{b}$ (blue curve).

However, such asymmetric dissipation does not lift CPA for $v_{a} \neq v_{b}$ but $v_{a}-v_{b} \approx 0$ and the CPA. Indeed, condition Eq. (5) of the main text gains a prefactor,

$$
\gamma_{*}=\frac{v_{a}+v_{b}}{2 v_{a} v_{b}} 8 \sin k
$$

as shown in Fig. 8.

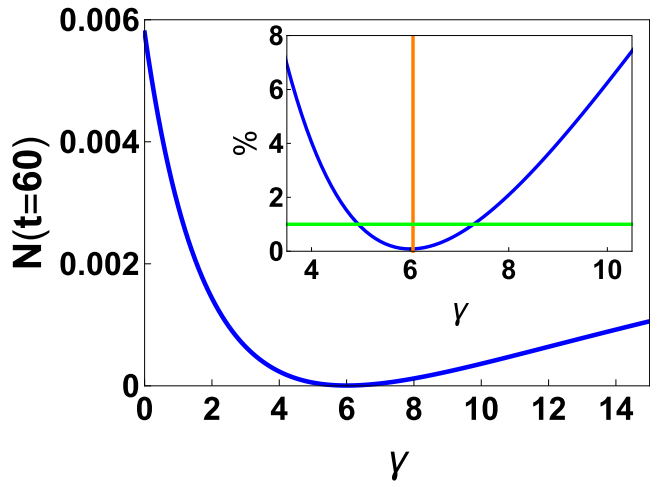

FIG. 8. Total norm $N$ at $t=60$ versus $\gamma$ for $v_{a}=1$ and $v_{b}=1.25$. Inset: Percentage (\%) at $t=60$, with $\gamma_{*}$ (orange); $1 \%$ of the norm (green).

This can be understood by employing a unitary transformation as in Ref. [11],

$$
\begin{array}{lll}
p_{n}=\frac{1}{\sqrt{2}}\left(a_{n}+b_{n}\right), & q_{n}=\frac{1}{\sqrt{2}}\left(a_{n}-b_{n}\right) \\
v_{+}=\frac{1}{2}\left(v_{a}+v_{b}\right), & v_{-}=\frac{1}{2}\left(v_{a}-v_{b}\right),
\end{array}
$$

which maps Eq. (A16) to

$$
\begin{aligned}
& \mu p_{n}=\frac{i \gamma}{2} v_{+} \delta_{n, 0} p_{0}-h p_{n}+\frac{i \gamma}{2} v_{-} \delta_{n, 0} q_{0}-2\left(p_{n+1}+p_{n-1}\right) \\
& \mu q_{n}=\frac{i \gamma}{2} v_{+} \delta_{n, 0} q_{0}+h q_{n}+\frac{i \gamma}{2} v_{-} \delta_{n, 0} p_{0} .
\end{aligned}
$$

For $v_{a}=v_{b}$, then $v^{-}=0$, which fully decoupled the two sublattices. The $p_{n}$ sublattice in Eq. (A19) yields the CPA condition $\gamma_{*}=8 \sin k$ in Eq. (A15). For $v_{a} \neq v_{b}$ with $v_{a}$ $v_{b} \approx 0$ will not largely renormalize $\mu$ in the $q_{n}$ sublattice, and hence at $n=0$ we approximate $\mu \approx h$. It then follows that $q_{0}=-\frac{v^{-}}{v^{+}} p_{0}$, which substituted into the $p_{n}$ sublattice yields

$$
\mu p_{n}=\frac{i \gamma}{2}\left(v_{+}-\frac{v_{-}^{2}}{v_{+}}\right) p_{0} \delta_{n, 0}-h p_{n}-2\left(p_{n+1}+p_{n-1}\right) .
$$

The prefactor of the dissipative strength $\gamma$ between brackets can be recast into

$$
\begin{aligned}
\frac{v_{+}^{2}-v_{-}^{2}}{v_{+}} & =\frac{2}{v_{a}+v_{b}} \frac{v_{a}^{2}+v_{b}^{2}+2 v_{a} v_{b}-\left(v_{a}^{2}+v_{b}^{2}-2 v_{a} v_{b}\right)}{4} \\
& =\frac{2 v_{a} v_{b}}{v_{a}+v_{b}}
\end{aligned}
$$

and it ultimately enters the CPA condition in Eq. (A17).

c. Case 2: $V_{2}$. The second case follows the former one, although with a different identity $\left[\mu_{1} \mathbb{I}_{2}+H_{0}+i \frac{\gamma}{2} V_{2}\right] \Phi_{1}=$ $\left[-4 \cos k+\frac{i \gamma}{2}(1+\delta)\right] \Phi_{1}$ employed in Eq. (A12). It then follows

$$
\begin{aligned}
U_{0}= & -\frac{2}{-4 \cos k+i \frac{\gamma}{2}(1+\delta)} \\
& \times\left[\alpha_{R} e^{i k}+\beta_{R} e^{-i k}+\alpha_{L} e^{-i k}+\beta_{L} e^{i k}\right] \Phi_{1} .
\end{aligned}
$$


A similar procedure leads to the transfer matrix entrees

$$
\begin{array}{ll}
M_{11}=\frac{8 \sin k-\gamma(1+\delta)}{8 \sin k} \mathbb{I}_{2}, & M_{12}=\frac{-\gamma(1+\delta)}{8 \sin k} \mathbb{I}_{2} \\
M_{21}=\frac{\gamma(1+\delta)}{8 \sin k} \mathbb{I}_{2}, & M_{22}=\frac{8 \sin k+\gamma(1+\delta)}{8 \sin k} \mathbb{I}_{2} .
\end{array}
$$

Imposing $M_{11}(k)=\mathbb{O}_{2}$ yields the CPA condition

$$
\gamma_{*}=\frac{8}{1+\delta} \sin k \Leftrightarrow k_{*}=\arcsin \frac{\gamma(1+\delta)}{8} .
$$

\section{Cross-stitch: Nonlinear regime}

In the nonlinear regime of the cross-stitch lattice we cannot apply the transfer matrix $M$ strategy in Eq. (A4) to obtain the CPA condition,

$$
\begin{aligned}
\mu a_{n}= & -a_{n-1}-a_{n+1}-b_{n-1}-b_{n+1}-h b_{n}+g\left|a_{n}\right|^{2} a_{n} \\
& -\left[\frac{i \gamma}{2} a_{n}+\frac{i \gamma \delta}{2} b_{n}\right] \delta_{n, 0} \\
\mu b_{n}= & -b_{n-1}-b_{n+1}-a_{n-1}-a_{n+1}-h a_{n}+g\left|b_{n}\right|^{2} b_{n} \\
& -\left[\frac{i \gamma \delta}{2} a_{n}+\frac{i \gamma}{2} b_{n}\right] \delta_{n, 0} .
\end{aligned}
$$

Nevertheless, we can show that nonlinearity does not alter the linear CPA condition in the following way. We consider only Case $2\left(V_{2}\right)$ of the dissipation, as this turns into $V_{1}$ for $\delta=0$.

Let us consider the steady-state solution $\Psi_{n}=$ $\left(a_{n}, b_{n}\right)^{T} e^{-i \mu t}$ for Eq. (A25). We then consider the ansatz Eq. (A1) reduced to $a_{L}=b_{R}=\rho \Phi_{1}$ and $\Phi_{1}=\frac{1}{\sqrt{2}}(1,1)^{T}$ and $b_{L}=a_{R}=0$,

$$
U_{n}=\Phi_{1} \begin{cases}\rho e^{i k n} & n<0 \\ \rho & n=0 \\ \rho e^{-i k n} & n>0\end{cases}
$$

For small amplitude waves, this fixes $\mu^{\prime}=-h-4 \cos k+$ $g \rho^{2}$. Equation (A25) at $n=0$ then reads

$$
\begin{aligned}
& \mu^{\prime} \rho=-2 \rho\left(e^{-i k}+e^{-i k}\right)-h \rho+g \rho^{3}-i \frac{\gamma}{2}(1+\delta) \rho \\
& \Leftrightarrow \mu^{\prime}=-4 e^{-i k}-h+g \rho^{2}-i \frac{\gamma}{2}(1+\delta) \\
& \Leftrightarrow-h-4 \cos k+g \rho^{2}=-4 e^{-i k}-h+g \rho^{2}-i \frac{\gamma}{2}(1+\delta) \\
& \Leftrightarrow-4 \cos k=-4 e^{-i k}-i \frac{\gamma}{2}(1+\delta) \\
& \Leftrightarrow \cos k-e^{-i k}=i \frac{\gamma}{8}(1+\delta) \\
& \Leftrightarrow \frac{e^{i k}-e^{-i k}}{2}=i \frac{\gamma}{8}(1+\delta) \\
& \Leftrightarrow \sin k=\frac{\gamma(1+\delta)}{8},
\end{aligned}
$$
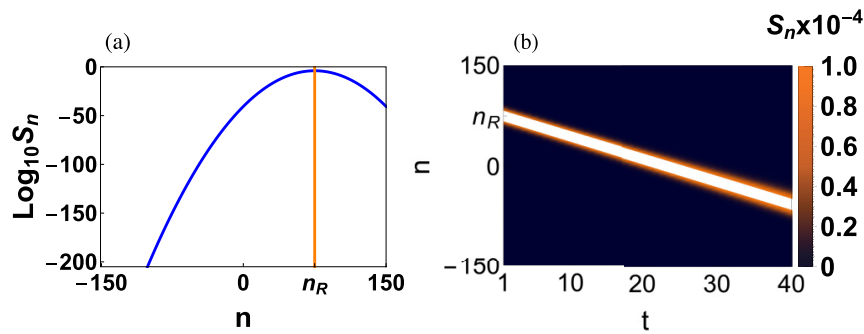

FIG. 9. (a) Spatial profile of the initial excitation in Eq. (B1) for $P_{0}=0.01$ and $\alpha=0.0075$, with $n_{R}=75$. (b) Time evolution of $S_{n}$ for $k=1$. Here $g=1, h=1$.

yielding the same CPA condition Eq. (A24) valid in the linear regime $g=0$.

\section{APPENDIX B: NUMERICAL SIMULATION}

\section{Gaussian beams}

Let us consider the cross-stitch lattice in Eq. (A25) written in components $\Psi_{n}=\left(a_{n}, b_{n}\right)^{T}$ with no dissipation $\gamma=0$. Since the mode $\Phi_{1}=\frac{1}{\sqrt{2}}(1,1)^{T}$ associated to the dispersive band of the model $\mu_{1}(k)=-h-4 \cos k$ is symmetric on the two components, we initialize a small-amplitude propagating wave with Gaussian profile located at the unit-cell $n_{R} \gg 0$ as shown in Fig. 9(a)-by defining a symmetric excitation $a_{n}=b_{n}$, with

$$
a_{n}=P_{0} e^{-\alpha\left(n-n_{R}\right)^{2}} e^{-i k\left(n-n_{R}\right)} .
$$

Here $k$ is the quasimomentum, $\alpha$ is called the inverse width of the wave and $P_{0}$ is the amplitude. In Fig. 9(b) we show the time evolution of this Gaussian beam. Let us observe that this wave packet is not monochromatic. However, the choice of $\alpha$ implies a spatial width of the beam of approximatively 15 unit-cells, with a correspondent frequency range of the packet in the reciprocal $k$ space lower than $10^{-1}$. This choice therefore keeps the frequency width of the wave packet close to the desired one.

In order to achieve CPA, two counterpropagating beams which meet at unit-cell $n=0$ (where the dissipation will be located) are required. We achieve this by considering two equal beams in Eq. (B1) which departs from opposite sides $n_{R} \gg 0$ and $n_{L} \ll 0$ with $n_{L}=-n_{R}$ and opposite quasimomentum $k$-as shown in Fig. 10(a)—by defining a symmetric
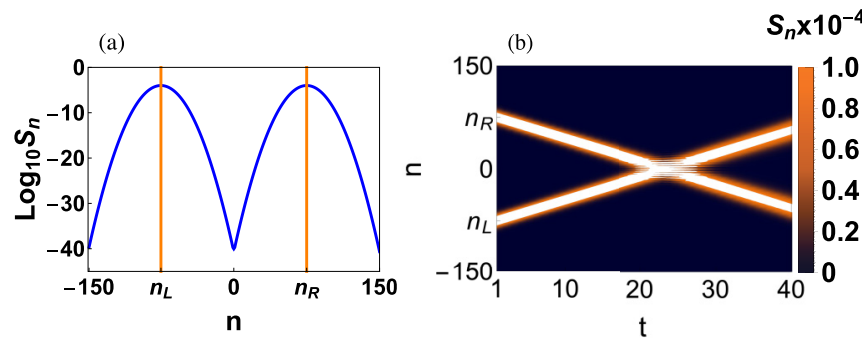

FIG. 10. (a) Spatial profile of the initial excitation in Eq. (B2) for $P=0.01$ and $\alpha=0.0075$, with $n_{R}=75$ and $n_{L}=-75$. (b) Time evolution of $S_{n}$ for $k=1$. Here $g=1, h=1$. 
excitation $a_{n}=b_{n}$ with

$$
a_{n}=P_{0} e^{-\alpha\left(n-n_{R}\right)^{2}} e^{-i k\left(n-n_{R}\right)}+P_{0} e^{-\alpha\left(n-n_{L}\right)^{2}} e^{i k\left(n-n_{L}\right)} .
$$

This is visualized in Fig. 10(b), where we see that the two beams collide at the unit-cell $n=0$.

The very same strategy has been applied to visualize CPA in the nonlinear diamond chain, whose components are $\Psi_{n}=\left(a_{n}, b_{n}, c_{n}\right)^{T}$. Since the eigenmodes $\phi_{1,2}=$ $\left(1,1, \pm \sqrt{2} e^{-k / 2}\right)^{T} / 2$ of the two dispersive bands $\mu_{1,2}(k)=$ $\left(-h \pm \sqrt{h^{2}+16+16 \cos k}\right) / 2$ is symmetric on the outer components $a_{n}, b_{n}$ and has $\pm \sqrt{2} e^{-k / 2}$ as a multiplicative factor on the central component $c_{n}$, we define the Gaussian beams

$$
a_{n}=P_{0} e^{-\alpha\left(n-n_{R}\right)^{2}} e^{-i k\left(n-n_{R}\right)}+P_{0} e^{-\alpha\left(n-n_{L}\right)^{2}} e^{i k\left(n-n_{L}\right)}
$$

with $b_{n}=a_{n}$ and $c_{n}= \pm \sqrt{2} e^{-k / 2} a_{n}$.

\section{Computations}

All the numerical simulations of the time evolution herewith shown have been performed using the commercial software Mathematica and employing the fourth-order explicit Runge-Kutta scheme.
[1] A. Mostafazadeh, Spectral Singularities of Complex Scattering Potentials and Infinite Reflection and Transmission Coefficients at Real Energies, Phys. Rev. Lett. 102, 220402 (2009).

[2] D. G. Baranov, A. Krasnok, T. Shegai, A. Alù, and Y. Chong, Coherent perfect absorbers: Linear control of light with light, Nat. Rev. Mater. 2, 17064 (2017).

[3] Y. D. Chong, L. Ge, H. Cao, and A. D. Stone, Coherent Perfect Absorbers: Time-Reversed Lasers, Phys. Rev. Lett. 105, 053901 (2010).

[4] S. Longhi, $\mathcal{P} \mathcal{T}$-symmetric laser absorber, Phys. Rev. A 82, 031801(R) (2010)

[5] Y. D. Chong, L. Ge, and A. D. Stone, $\mathcal{P} \mathcal{T}$-Symmetry Breaking and Laser-Absorber Modes in Optical Scattering Systems, Phys. Rev. Lett. 106, 093902 (2011).

[6] D. A. Zezyulin, H. Ott, and V. V. Konotop, Coherent perfect absorber and laser for nonlinear waves in optical waveguide arrays, Opt. Lett. 43, 5901 (2018).

[7] A. Müllers, B. Santra, C. Baals, J. Jiang, J. Benary, R. Labouvie, D. A. Zezyulin, V. V. Konotop, and H. Ott, Coherent perfect absorption of nonlinear matter waves, Sci. Adv. 4, eaat6539 (2018).

[8] J. Jeffers, Nonlocal Coherent Perfect Absorption, Phys. Rev. Lett. 123, 143602 (2019).

[9] D. Leykam, A. Andreanov, and S. Flach, Artificial flat band systems: From lattice models to experiments, Adv. Phys.: X 3, 1473052 (2018).

[10] D. Leykam and S. Flach, Perspective: Photonic flatbands, APL Photon. 3, 070901 (2018).

[11] S. Flach, D. Leykam, J. D. Bodyfelt, P. Matthies, and A. S. Desyatnikov, Detangling flat bands into Fano lattices, Europhys. Lett. 105, 30001 (2014).

[12] W. Maimaiti, A. Andreanov, H. C. Park, O. Gendelman, and S. Flach, Compact localized states and flat-band generators in one dimension, Phys. Rev. B 95, 115135 (2017).

[13] M. Röntgen, C. V. Morfonios, and P. Schmelcher, Compact localized states and flat bands from local symmetry partitioning, Phys. Rev. B 97, 035161 (2018).

[14] W. Maimaiti, S. Flach, and A. Andreanov, Universal $d=1$ flat band generator from compact localized states, Phys. Rev. B 99, 125129 (2019).

[15] D. Leykam, S. Flach, O. Bahat-Treidel, and A. S. Desyatnikov, Flat band states: Disorder and nonlinearity, Phys. Rev. B 88, 224203 (2013).

[16] J. D. Bodyfelt, D. Leykam, C. Danieli, X. Yu, and S. Flach, Flatbands under Correlated Perturbations, Phys. Rev. Lett. 113, 236403 (2014).
[17] C. Danieli, J. D. Bodyfelt, and S. Flach, Flat-band engineering of mobility edges, Phys. Rev. B 91, 235134 (2015).

[18] D. Leykam, J. D. Bodyfelt, A. S. Desyatnikov, and S. Flach, Localization of weakly disordered flat band states, Eur. Phys. J. B 90, 1 (2017).

[19] M. Johansson, U. Naether, and R. A. Vicencio, Compactification tuning for nonlinear localized modes in sawtooth lattices, Phys. Rev. E 92, 032912 (2015).

[20] A. Ramachandran, C. Danieli, and S. Flach, Fano resonances in flat band networks, in Fano Resonances in Optics and Microwaves: Physics and Applications, edited by E. Kamenetskii, A. Sadreev, and A. Miroshnichenko (Springer International Publishing, Cham, 2018), pp. 311-329.

[21] C. Danieli, A. Maluckov, and S. Flach, Compact discrete breathers on flat-band networks, J. Low Temp. Phys. 44, 678 (2018).

[22] S. Mukherjee, A. Spracklen, D. Choudhury, N. Goldman, P. Öhberg, E. Andersson, and R. R. Thomson, Observation of a Localized Flat-Band State in a Photonic Lieb Lattice, Phys. Rev. Lett. 114, 245504 (2015).

[23] R. A. Vicencio, C. Cantillano, L. Morales-Inostroza, B. Real, C. Mejía-Cortés, S. Weimann, A. Szameit, and M. I. Molina, Observation of Localized States in Lieb Photonic Lattices, Phys. Rev. Lett. 114, 245503 (2015).

[24] S. Weimann, L. Morales-Inostroza, B. Real, C. Cantillano, A. Szameit, and R. A. Vicencio, Transport in sawtooth photonic lattices, Opt. Lett. 41, 2414 (2016).

[25] N. Masumoto, N. Y. Kim, T. Byrnes, K. Kusudo, A. Löffler, S. Höfling, A. Forchel, and Y. Yamamoto, Exciton-polariton condensates with flat bands in a two-dimensional kagome lattice, New J. Phys. 14, 065002 (2012).

[26] S. Taie, H. Ozawa, T. Ichinose, T. Nishio, S. Nakajima, and Y. Takahashi, Coherent driving and freezing of bosonic matter wave in an optical Lieb lattice, Sci. Adv. 1, e1500854 (2015).

[27] R. A. Vicencio, J. Brand, and S. Flach, Fano Blockade by a Bose-Einstein Condensate in an Optical Lattice, Phys. Rev. Lett. 98, 184102 (2007).

[28] D. Leykam, S. Flach, and Y. D. Chong, Flat bands in lattices with non-Hermitian coupling, Phys. Rev. B 96, 064305 (2017).

[29] J. Vidal, B. Douçot, R. Mosseri, and P. Butaud, Interaction Induced Delocalization for Two Particles in a Periodic Potential, Phys. Rev. Lett. 85, 3906 (2000).

[30] M. Di Liberto, S. Mukherjee, and N. Goldman, Nonlinear dynamics of Aharonov-Bohm cages, Phys. Rev. A 100, 043829 (2019). 
[31] G. Gligorić, P. P. Beličev, D. Leykam, and A. Maluckov, Nonlinear symmetry breaking of Aharonov-Bohm cages, Phys. Rev. A 99, 013826 (2019).

[32] R. Khomeriki and S. Flach, Landau-Zener Bloch Oscillations with Perturbed Flat Bands, Phys. Rev. Lett. 116, 245301 (2016).

[33] S. Mukherjee and R. R. Thomson, Observation of localized flat-band modes in a quasi-one-dimensional photonic rhombic lattice, Opt. Lett. 40, 5443 (2015).

[34] S. Mukherjee and R. R. Thomson, Observation of robust flatband localization in driven photonic rhombic lattices, Opt. Lett. 42, 2243 (2017).

[35] S. Mukherjee, M. Di Liberto, P. Öhberg, R. R. Thomson, and N. Goldman, Experimental Observation of Aharonov-Bohm Cages in Photonic Lattices, Phys. Rev. Lett. 121, 075502 (2018).

[36] M. Niţă, B. Ostahie, and A. Aldea, Spectral and transport properties of the two-dimensional Lieb lattice, Phys. Rev. B 87, 125428 (2013).

[37] J.-P. Eckmann and C. E. Wayne, Decay of Hamiltonian breathers under dissipation, arXiv:1907.12632.
[38] A. S. Rodrigues, K. Li, V. Achilleos, P. G. Kevrekidis, D. J. Frantzeskakis, and C. M. Bender, $\mathcal{P} \mathcal{T}$-symmetric double well potentials revisited: Bifurcations, stability and dynamics, arXiv:1207.1066.

[39] C. W. Hsu, B. Zhen, D. A. Stone, J. D. Joannopoulos, and M. Soljacic, Bound states in the continuum, Nat. Rev. Mater. 1, 16048 (2016).

[40] X. Fang, M. Lun Tseng, J.-Y. Ou, K. F. MacDonald, D. Ping Tsai, and N. I. Zheludev, Ultrafast all-optical switching via coherent modulation of metamaterial absorption, Appl. Phys. Lett. 104, 141102 (2014).

[41] W. Wan, Y. Chong, L. Ge, H. Noh, A. D. Stone, and H. Cao, Time-reversed lasing and interferometric control of absorption, Science 331, 889 (2011).

[42] M. Papaioannou, E. Plum, J. Valente, E. T. F. Rogers, and N. I. Zheludev, All-optical multichannel logic based on coherent perfect absorption in a plasmonic metamaterial, APL Photon. 1, 090801 (2016). 